Ann. Zootech., I966, 15 (4), 367-37I.

\title{
RELATION ENTRE LA TENEUR DU MUSCLE DE PORC EN DESMO- ET LYO-GLYCOGÈNES ET L'INTENSITÉ DE LA GLYCOGENOLYSE POST MORTEM
}

\author{
J. CHARPENTIER \\ avec la collaboration technique de Denise Guène et de J.-P. Suquet \\ Laboratoire de Recherches sur la Viande, \\ Centre national de Recherches zootechniques, 78-Jouy-en-Josas
}

SOMMAIRE

La teneur en glycogène ainsi que l'importance relative de la forme "liée " (desmoglycogène) et de la forme "libre " (lyoglycogène) ont été déterminées dans les muscles Semi tendinosus et Lon. gissimus dorsi de 20 porcs Large White, d'une part, sur des échantillons prélevés immédiatement après l'abattage et, d'autre part, sur des échantillons maintenus en anaérobiose à $37^{\circ} \mathrm{C}$ pendant une heure.

L'intensité de la glycogénolyse pendant la première heure post-mortem, qui conditionne en partie la rétention d'eau et la couleur du tissu musculaire, est plus importante dans les muscles présentant une forte teneur en glycogène "lié ".

Depuis les travaux de WILISTÄTER et ROHDEWALD (I934), de nombreux auteurs ont admis que le glycogène existe dans les tissus sous deux formes : 1'une facilement extractible par l'acide trichloracétique, existe à l'état libre dans la cellule, l'autre, probablement liée à des protéines, ne peut être extraite que par la potasse à chaud (BLOOM et al., I95I ; KITS et KEMP, I955; RUSSEL et BLOOM, I955 ; STETTTEN, Katzen et StetTen, I958; Loureau et MEyer, I960; KUgler et Wilkinson, i96o; NEUzI, I964). La première fraction est connue sous le nom de glycogène libre ou "lyoglycogène ". La seconde est désignée par le terme de glycogène fixé ou " desmoglycogène ".

Toutefois plusieurs auteurs (CAROI, L, LONGLEY et ROE, I956; MEYER et ZaLTA, I958; HANSON, SchWARTZ et BARKER, I960) contestent la validité de la distinction entre fraction libre et fixée dans la mesure oû l'importance relative de la fraction libre dépend du degré de désintégration mécanique des tissus obtenu lors du processus d'extraction. Cependant lorsqu'une technique identique est utilisée, les deux fractions sont affectées d'une façon très différente par de nombreux facteurs physiologiques (BLoOM et al., I95I). Ainsi dans le cas de la glycogénolyse musculaire, l'utilisation prépondérante de l'une ou l'autre des fractions en fonction de diverse $\mathrm{S}_{\mathrm{S}}$ 
conditions expérimentales, a pu être mise en évidence (BLoOm et al., I95I; BLoom et KNowitor I953).

En ce qui concerne la glycogénolyse post mortem, dont 1'intensité et la rapidité conditionnent très fortement la valeur de nombreuses caractéristiques physicochimiques du tissu musculaire, l'évolution des fractions libres et fixées n'a fait, à notre connaissance, l'objet d'aucune étude systématique. WISMER-PEDERSEN et BRISkey (I96I) ont toutefois mentionné l'importance plus grande de la fraction fixée dans les muscles de porc présentant une chute de $\mathrm{pH}$ rapide post mortem Aussi nous sommes-nous proposés d'étudier l'évolution post mortem du glycogène libre et du glycogène fixé dans lè cas du muscle de porc, lequel présente précisément l'intérêt de manifester une très grande variabilité dans la rapidité de la glycogénolyse post mortem.

\section{I. - MATÉRIEL E'T MÉTHODES}

Des échantillons, d'un poids dé $100 \mathrm{~g}$ environ, étaient prélevés, sitôt la saignée terminée, sur les muscles Semi tentinosus et Longissimus dorsi de 20 porcs Large White pesant entre 100 et I Io kg. Des parties aliquotes, d'un poids compris entre 5 et IO g, étaient immédiatement prélevées sur ces échantillons et faisaient l'objet de déterminations de glycogène libre et fixé. Le reliquat des échantillons était maintenu à $30^{\circ} \mathrm{C}$ sous atmosphère d'azote pendant une heure. (Nous avons retenu une durée de glycogénolyse d'une heure, car dans le cas de muscles présentant une évolution post mortem du $\mathrm{pH}$ particuliercment rapide, le pll ultime est effectivement atteint une heure apres la mort).

Les teneur en glycogène libre et en glycogène fixé étaient de nouveau déterminées 1 heure post morlem.

Le glycogène libre était dosé par la méthode de BLon et al. (1951) après brovage du tissu musculaire à í 000 tours/minute pendant une minute dans une solution de TC: 10 p. 100 à l'aide d'un broyeur Biorex. Le glycogène total était déterminé après digestion dans la potasse à .,5 p. 100 par la néthode de (iood, Kramer. Somogyi (193.3).

Le glycogène fisé correspondait à la différence entre ke glycogène total et le glycogène libre.

\section{II. — RÉSUITATS ET DISCUSSION}

Le tableau I montre que, d'une part, la variabilité des teneurs en glycogène fixé est du même ordre que celle des teneurs en glycogène libre et que, d'autre part,

\section{TABLEAU I}

Valeurs moyennes des teneurs en glycogène total, libre et fixé des muscles Semi-tendinosus et Longissinus dorsi au temps séro et après une heure en anaérobiose à $30^{\circ} \mathrm{C}$ ( $\mu g$ de glycogène pour $100 \mathrm{mg}$ de tissu frais)

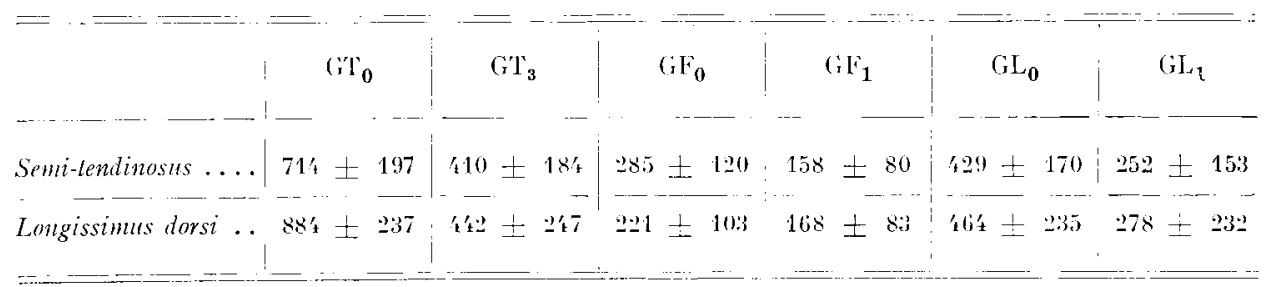

la diminution de la teneur en glycogène total porte à la fois sur les fractions libres et fixées. 
Les valeurs des coefficients de corrélation mentionnées dans le tableau 2 montrent que pendant la première heure post mortem :

TABLEAU 2

Liaison entre la quantité de glycogène total disparu pendant la première heure past mortem en anaérobiose à $30^{\circ} \mathrm{C}$ et les teneurs initiales en glycogène total, libre et fixé

\begin{tabular}{c|c|c|c}
\hline \hline $\begin{array}{c}\text { Coefficients } \\
\text { de corrélation } \\
\text { entre } \\
\left(\mathrm{GT}_{\mathbf{0}^{-}} \mathrm{GT}_{\mathbf{1}}\right) \text { et }\end{array}$ & \begin{tabular}{c} 
Muscles \\
\cline { 2 - 4 } \\
\hline $\mathrm{GT}_{\mathbf{0}}$
\end{tabular} & $\begin{array}{c}\text { Songissimus dorsi } \\
\text { Semi-tendinosus }\end{array}$ & $\begin{array}{c}\text { Semi-tendinosus } \\
+ \text { Longissimus dorsi } \\
(2)\end{array}$ \\
\hline $\mathrm{GL}_{\mathbf{0}}$ & $+0,51$ & $+0,23$ & $+0,39$ \\
\hline $\mathrm{GF}_{\mathbf{0}}$ & $+0,10$ & $+0,0^{\prime}$ & $+0,05$ \\
\hline
\end{tabular}

(1) seuil de signification à $\mathrm{P} 0,01=0,5^{\prime} \mathrm{x}-$ et $-\mathrm{P} 0,05=0, \mathbf{t}^{3}$

(2) - $\quad$ à $\mathrm{P} 0,01=0,41 \ldots$ et $-\mathrm{P} 0,05=0,32$

$\mathrm{GT}_{0}=$ Glycogène total au temps zéro

$\mathrm{GF}_{0}=\quad$ fixé -

$\mathrm{GT}_{1}=$ Glycogène total 1 heure post mortem

$\mathrm{GL}_{\mathbf{0}}=-$ libre -

$\begin{array}{lll}\mathrm{GF}_{1}= & \text { fixé } & - \\ \mathrm{GL}_{1}= & - & \text { libre }\end{array}$

I. L'intensité de la glycogénolyse ne semble pas étroitement liée à l'importance de la teneur en glycogène du muscle au moment de l'abattage, puisque les valeurs des coefficients de corrélation ne sont significatives au seuil de $5 \mathrm{p}$. Ioo que dans le cas du muscle Semi tendinosus et également pour l'ensemble des muscles Semi tendinosus et Longissimus dorsi considérés en bloc. Ces résultats confirment ceux de Wismer-Pedersen et Briskey (I96I). Selon ces auteurs, en effet, la vitesse de chute du $\mathrm{pH}$ post mortem dans le muscle de porc n'est pas systématiquement liée à la quantité de glycogène présente dans le muscle au moment de l'abattage.

2. L'intensité de la glycogénolyse est, par contre, liée assez étroitement à l'importance de la teneur en glycogène fixé. Ce résultat confirme pleinement les observations de WISMER-PEDERSEN et BRISKEY (I96I) qui concluaient à l'existence vraisemblable d'une fraction fixée plus importante dans le cas de muscles présentant une chute de $\mathrm{pH}$ post mortem plus rapide. Il est à noter que, selon certains auteurs, le glycogène fixé apparaît comme métaboliquement plus actif. Ainsi, d'une part, après injection de glucose $\mathrm{C}_{14}$ à des rats, DRATZ, RUSSEL et Cover, (I956), STETTEN, KATZEN et STETTEN (I958), Loureau et MEyer (I960) constatent que les activités spécifiques de la fraction fixée sont nettement plus élevées. D'autre part, il convient de noter également que de nombreux enzymes glycolytiques sont liés aux protéines myofibrillaires (AMBERSON, ROISEN et BAUER, I965).

Il semble plausible d'émettre 1'hypothèse selon laquelle le glycogène fixé, métaboliquement plus actif, serait plus important dans les muscles pour lesquels les processus glycolytiques anaérobies sont prédominants. 


\section{CONCLUSION}

La variabilité des teneurs en glycogène libre et glycogène fixé du muscle de porc est importante.

Il existe une liaison très nette entre l'importance de la fraction fixée et l'intensité de la glycogénolyse pendant la première heure post mortem. Ce résultat semble contribuer à rendre plausible l'hypothèse selon laquelle les glycogènes " libre " et " fixé " dont l'existence effective fait encore l'objet de controverses, représenteraient en fait de véritables entités physiologiques.

En outre, d'un point de vue technologique, compte tenu des répercussions importantes de la vitesse de glycogénolyse post mortem sur diverses caractéristiques du tissu musculaire, en particulier la capacité de rétention d'eau, ce résuitat montre également que la structure du glycogène est un élément important à considérer dans l'étiologie du muscle exsudatif.

Reçu pour publication en décembre 1966.

\section{SUMMARY}

RELATION OF CONTENTS OF DESMO- AND LYO-GLYCOGEN IN MUSCLE OF THE PIG TO RATE OF BREAKDOWN OF GLYCOGEN "POST MORTEM "

In pig muscle the rate of anaerobic breakdown of glycogen post mortem varies greatly.

Of the many causes which could explain this variability it seems logical to consider, among others, eventual changes in the structure of the glycogen, notably in regard to the degree to which glycogen is bound to the muscle proteins.

For this purpose the glycogen content, and also the relative proportions of the bound form, desmoglycogen, and the free form, lyoglycogen, were estimated in semi tendinosus and longissimus dorsi muscles of 20 Large White pigs. Estimations were made on samples taken immediately after slaughter and in samples kept in anaerobic conditions at $30^{\circ} \mathrm{C}$ for one hour.

The results of the experiment show that intensity of breakdown of glycogen post mortem is greater in the muscles with a high content of bound glycogen (table. 2).

\section{RÉFÉRENCES BIBLIOGRAPHIQUES}

Amberson W. R., Roisen F. J., Bauer A. C., ig65. The attachment of glycolytic enzymes to muscle ultrastructure. J. cell. comp. Physiol., 66, $7 \mathrm{I}-90$.

Bloom W. L., Lewis G. T., Schumpert H. Z., Shex T., I95I. Glycogen fractions of liver and muscle. J. biol. Chem., 177, 631-636.

Bloom W. L., Knowlton G. C., 1953. Muscle glycogen fractions during stimulation. Amer. J. Physiol., $173,545-546$.

Blount D. H., Mever D. K., 1959. Effects of cardiac work on glycogen fractions of the heart. Amer. J. Physiol., 197, ror 3-1016.

Caroll N. V., Longley R. W., Roe J. H., 1956. The determination of glycogen in liver and muscle by use of anthrone reagent. J. biol. Chem., 220, $583-593$.

Cordier D., Dessalx G., 1952. Variation des diverses formes du glycogène cardiaque sous l'influence d'une anoxie de longue duree. J. Physiol., 44, 703-707.

Dratz A. F., Russel J. A., Covey B. M., I956. Liptake of labeled glucose by tissue glycogen in vivo. Fed. Procesd., 15, 52 . 
Goov C. A., Kramer H., Sonogy M., I933. The determination of glycogen. J. biol. Chem., 100, 485 . HaNson R. W., Schwartz H. S., Barker S. B., ig6o. "Free" and fixed " glycogen as physiologicial entities. Amer. J. Physiol., 198, 800-806.

Kits A. J. H., Kemp A., 1955. Free and fixed glycogen in rat muscle. Biochem. J., 59, 487.

Kugler J. H., Wilkinson W. J. C., I959. A relation between total glycogen content of ox myocardium and its histochemical demonstration. f. Hislochem. Cytochem., 7, 398-402.

Lolreat II., MIeyer F., ig6o. Nouvelles recherches sur l'incorporation dans le desmoglycogène et le lyoglycogène isolés du foie de rat du $\mathrm{C}_{\mathbf{1 4}}$ foumi par le glucose. J. Physiol., 52, 835-845.

Meyer F., Zalta J. P., 1958. Sur l'état du glycogène dans le foie du rat. C. R. Acad. Sci., 247, 357.

Neuzil E., I964. Données modernes concernant la structure et le métabolisme du glycogène. Pathol., Biol., 12, $209^{-2} \mathrm{I} 7$.

Russel J. A., Bцоом W. L., 1955. Hxtractable and residual glycogen in tissues of the rat. Amer. J. Physiol., 183, 345-355.

StetTey M. R., KaRzeN H. II., STETTEN D. J., I958. A comparison of the glycogen isolated by acid and alkaline procedures. $J . B$. C., 232, $475^{-487}$.

Willstäter R., Rohdewald M., 1934. Uber den Zustand des Glytrogens in der Leber, im Muskel und in Leukocyten. Hoppe Seyl. Z. physiol. Chem., 225, I03-124.

Wismer-Pedersen J., Briskey F. J., I96I. Rate of anaerobic glycolysis versus structure in pork muscle. Nature, 183, 318-320. 